\title{
MRI-guided cryoablation for metastatic spine disease: intermediate-term clinical outcomes in 14 consecutive patients
}

\author{
*Ziev B. Moses, MD, ${ }^{1}$ Thomas C. Lee, MD, ${ }^{2}$ Kevin T. Huang, MD, ${ }^{1}$ Jeffrey P. Guenette, MD, ${ }^{2}$ and \\ John H. Chi, MD, MPH ${ }^{1}$
} 1Department of Neurosurgery and 2Division of Neuroradiology, Brigham and Women's Hospital, Harvard Medical School,
Boston, Massachusetts

\begin{abstract}
OBJECTIVE Minimal access ablative techniques have emerged as a less invasive option for spinal metastatic disease reduction and separation from neural tissue. Compared with heat-based ablation modalities, percutaneous imageguided cryoablation allows for more distinct visualization of treatment margins. The authors report on a series of patients undergoing MRI-guided cryoablation as a feasible method for treating spinal metastatic disease.
\end{abstract}

METHODS A total of 14 patients with metastatic spine disease undergoing MR-monitored cryoablation were prospectively enrolled. Procedures were performed in an advanced imaging operating suite with the use of both CT and MRI to gain access to the spinal canal and monitor real-time cryoablation.

RESULTS The average age was 54.5 years (range $35-81$ years). The mean preoperative Karnofsky Performance Status score was 79.3 (range 35-90). The average radiographic follow-up was 7.1 months (range 25-772 days), and the average clinical follow-up was 9.8 months (range 7-943 days). In 10 patients with epidural disease, 7 patients underwent postprocedural imaging, and of these $71 \%(5 / 7)$ had stable or reduced radiographic disease burden. Bone regrowth was observed in $63 \%(5 / 8)$ of patients with bone ablation during the treatment who had postoperative imaging. Pre- and postoperative visual analog scale scores were obtained, and a significant reduction in these scores was found following ablation. There were no complications.

CONCLUSIONS MR-guided cryoablation is a minimally invasive treatment option for metastatic spine disease. In patients with epidural disease, the majority experienced tumor reduction or arrest at follow-up. In addition, pain was significantly improved following ablation. The average hospital stay was short, and the procedure was safe in a range of patients who are otherwise not ideal candidates for standard treatment.

https://thejns.org/doi/abs/10.3171/2019.11.SPINE19808

KEYWORDS spinal oncology; minimally invasive spine surgery; metastatic spine disease

$\mathrm{T}$ HE benefit of surgery in metastatic epidural spine disease was demonstrated in a randomized controlled study by Patchell et al., who found outcomes in patients with metastatic spine disease with epidural extension to be improved in measures such as incontinence, pain, and overall survival when undergoing decompression and stabilization followed by conventional radiation therapy compared with those undergoing radiation therapy alone. ${ }^{16}$ More recently, despite the advent of stereotactic radiosurgery for metastatic spine disease, epidural involvement often limits the allowable dose able to be delivered to the spine that can impact local disease control. "Separation surgery," as popularized by Bilsky and colleagues, whereby a smaller operation is performed to separate the epidural disease from the neuraxis, followed by spinal stabilization if needed, has been advocated as a way to maximize radiation therapy and improve local disease control. ${ }^{10}$ This allows for a higher dose of radiation and minimizes the odds of spinal cord toxicity. However, traditional open spine surgery is not without significant

ABBREVIATIONS KPS = Karnofsky Performance Status; RFA = radiofrequency ablation; SLITT = spinal laser interstitial thermal therapy; VAS = visual analog scale. SUBMITTED July 22, 2019. ACCEPTED November 13, 2019.

* Z.B.M. and T.C.L. contributed equally to this work. 
TABLE 1. Demographic and baseline data of 14 patients undergoing spinal cryoablation

\begin{tabular}{ccccccc}
\hline Case No. & $\begin{array}{c}\text { Age }(\mathrm{yrs}), \\
\text { Sex }\end{array}$ & $\begin{array}{c}\text { Tumor } \\
\text { Location }\end{array}$ & $\begin{array}{c}\text { Primary } \\
\text { Malignancy }\end{array}$ & $\begin{array}{c}\text { Preop KPS } \\
\text { Score }\end{array}$ & $\begin{array}{c}\text { No. of sLITT } \\
\text { Fibers }\end{array}$ & $\begin{array}{c}\text { Length of Stay } \\
\text { (days) }\end{array}$ \\
\hline 1 & $51, \mathrm{~F}$ & T10 & Lung & 90 & 1 & 1 \\
\hline 2 & $48, \mathrm{~F}$ & $\mathrm{~T} 12$ & Breast & 90 & 1 & 0 \\
\hline 3 & $59, \mathrm{M}$ & $\mathrm{T} 11$ & Renal & 90 & 5 & 0 \\
\hline 4 & $64, \mathrm{M}$ & Sacrum & Tonsil & 55 & 2 & 1 \\
\hline 5 & $35, \mathrm{M}$ & T6 & Lung & 90 & 2 & 1 \\
\hline 6 & $51, \mathrm{~F}$ & Sacrum & Thyroid & 80 & 2 & 0 \\
\hline 7 & $73, \mathrm{M}$ & T2 & Lung & 60 & 2 & 3 \\
\hline 8 & $47, \mathrm{~F}$ & T11 & Breast & 80 & 2 & 1 \\
\hline 9 & $81, \mathrm{~F}$ & T4 & Renal & 90 & 2 & 2 \\
\hline 10 & $65, \mathrm{~F}$ & T6-7 & Rectal & 90 & 1 & 0 \\
\hline 11 & $33, \mathrm{~F}$ & T4 & Lung & 35 & 2 & 12 \\
\hline 12 & $55, \mathrm{~F}$ & L5-S1 & Breast & 80 & 1 & 0 \\
\hline 13 & $36, \mathrm{M}$ & T4 & Melanoma & 90 & 1 & 0 \\
\hline 14 & $65, \mathrm{M}$ & C6-7 & Renal & 90 & 2 & 0 \\
\hline
\end{tabular}

risks and recovery from surgery can often delay the onset of radiation therapy.

More recently, using minimal access techniques, others have advocated for surgical separation of metastatic spinal disease from neural elements using spinal laser interstitial thermal therapy (sLITT).$^{19}$ This technique relies on real-time MR thermometry to monitor the heat zone and protect the neural elements. However, technical limitations of this technique include a limited ability to clearly visualize the edges of the heat zone with high-resolution imaging and poor intraprocedural temporal resolution. In the series by Tatsui et al., 1 patient developed spinothalamic tract dysfunction and postprocedural heat-related edema. ${ }^{19}$ Others have reported similar heat-related edema in the brain. ${ }^{13}$ We report on our experience using minimally invasive cryoablation to treat metastatic spinal disease.

\section{Methods}

\section{Patient Population}

A total of 14 patients (6 males and 8 females) with spine metastases were prospectively enrolled in a study of image-guided cryoablation for head, neck, and spine tumors (clinical trial registration no. NCT02085941 [clinicaltrials. gov]) between 2015 and 2018. Patients ranged in age from 35 to 81 years, with a mean age of 54.5 years. All patients had documented histological malignancy at a primary site outside the spine. See Table 1 for baseline demographic data. A multidisciplinary tumor board comprising radiation oncologists, radiologists, medical oncologists, neurosurgeons, and orthopedic surgeons reviewed all cases and made the decision for cryoablation. In general, patients were considered for inclusion if they had a spinal tumor, including epidural tumor, and it was felt unsafe to proceed with stereotactic radiosurgery or if they had advanced metastatic disease and had previously undergone radiation and were deemed too unfit for traditional surgical intervention. In addition, patients on active chemotherapy, where it was decided by the medical oncologist that treatment would need to stop for open surgery were considered for cryoablation. While no strict cutoffs for Karnofsky Performance Status (KPS) scores were used, patients with lower scores were favored for cryoablation, as it was often deemed unsafe for them to undergo open separation surgery. Patients with acute neurological findings or an unstable spine were excluded from treatment consideration. All patients were evaluated prior to treatment and at 1 month, 3 months, and 6 months following the procedure. Physical examination, visual analog scale (VAS) score, and followup imaging were obtained at these visits. Further followup and imaging were performed at the discretion of the primary oncology team. Epidural spinal cord compression (ESCC) scale scores were calculated in patients with applicable epidural tumor extension. ${ }^{4}$ In light of prior findings of bone regrowth following tumor cryoablation, this was also assessed on postablation imaging. ${ }^{12}$ This study was approved by the Partners Human Research Committee institutional review board, and all patients provided informed consent.

\section{Cryoablation Procedure}

All procedures were performed in an advanced multimodality imaging suite with patients under general endotracheal anesthesia. The patient is positioned prone on the CT table on gel rolls with the arms tucked. A selfadhesive fiducial grid is placed on the skin (Fig. 1C); the skin entry site is marked and the grid removed, and initial access to the epidural space is obtained using a bone drill (Arrow OnControl Power Driver, Teleflex) under CT guidance (Somatom Sensation 64, Siemens Healthcare). Cryoprobes (IceSeed, Galil Medical) are then placed under CT guidance down the drill tracks into the vertebral body and/or epidural space. The patient is then transported into the MRI scanner for cryoablation. The ice ball is monitored with serial 1-minute T2-weighted MRI (either axial T2-weighted turbo spin echo with TR 3000 msec, TE 87 

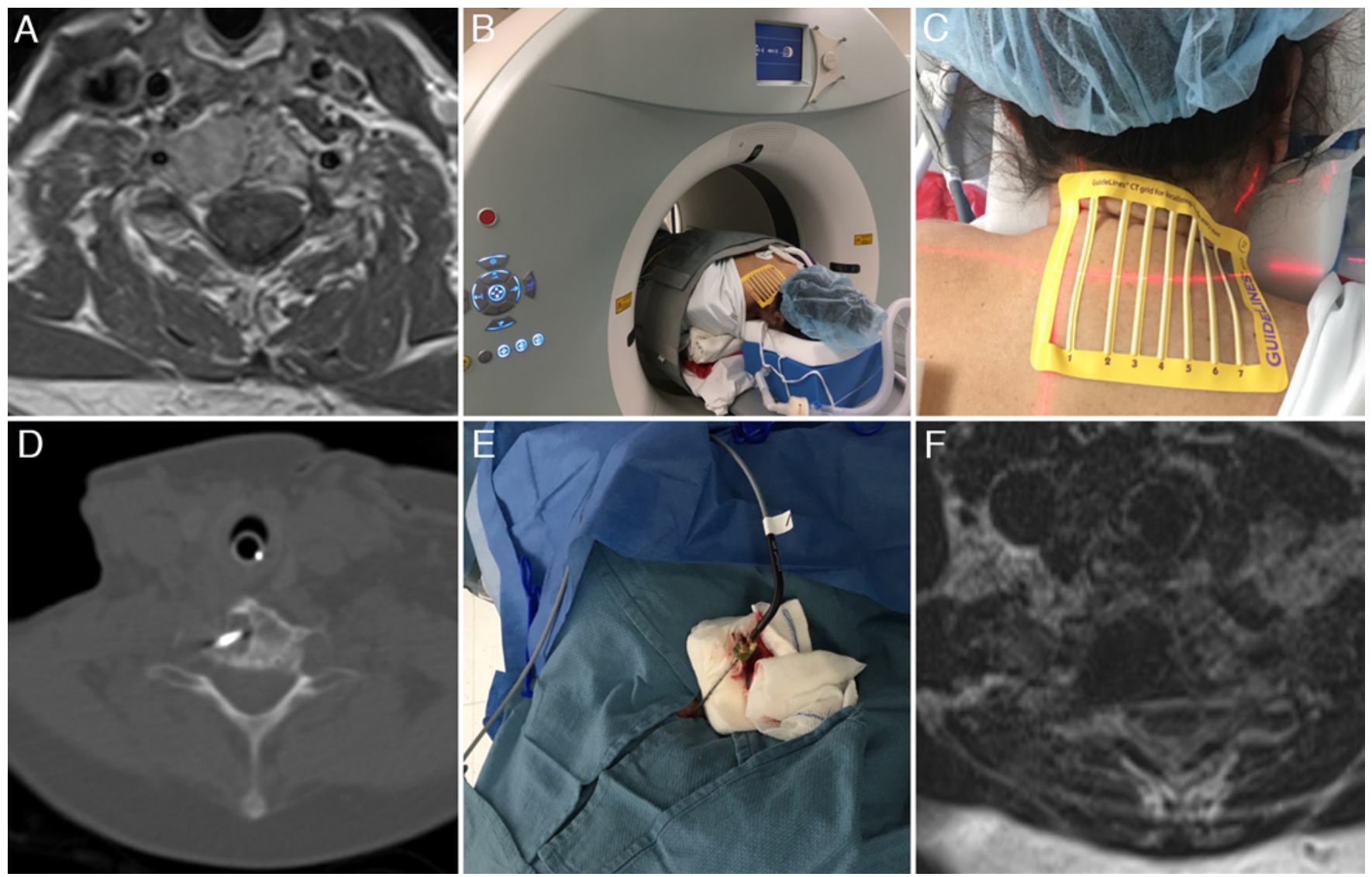

FIG. 1. A: Preoperative axial T2-weighted MR image demonstrating a vertebral body mass with epidural extension. B: Intraoperative photograph of a patient placed prone in the CT scanner for lesion targeting. C: Close-up view of the CT grid to assist in localization of the skin entry point. D: Intraoperative CT scan demonstrating successful placement of the drill in the mass. E: Depiction of the IceSeed cryoprobe placed through the power drill driver to the target. F: Intraoperative axial T2-weighted MR image of the ice ball during cryoablation. Figure is available in color online only.

msec, thickness $3 \mathrm{~mm}$; or axial T2-weighted BLADE with TR $2000 \mathrm{msec}$, TE $113 \mathrm{msec}$, thickness $4 \mathrm{~mm}$ ) using a 3-T MRI scanner (Magnetom Verio, Siemens Healthcare). ${ }^{9}$ Cryoablation is performed (SeedNet Cryoablation System, Galil Medical) in successive freeze-thaw cycles at variable powers and durations based on real-time MRI data. See Fig. 1 for an example depicting the standard workflow.

\section{Statistical Analysis}

A Wilcoxon signed-rank test was performed to assess for significance of VAS score changes before and after cryoablation. A paired Student t-test was used for comparing pre- and postablation epidural tumor thickness; $\mathrm{p}<$ 0.05 was considered significant.

\section{Results}

A variety of pathologies were treated, including lung cancer in 4 patients; renal cell cancer in 3 patients; breast cancer in 3 patients; and rectal cancer, thyroid cancer, tonsil cancer, and melanoma in 1 patient each. The most common region treated was the thoracic spine (10 patients), followed by the sacrum ( 2 patients) and the cervical and lumbar regions (1 patient each). All patients had under- gone some form of treatment for their metastatic spine disease prior to cryoablation, including 9 who had undergone chemotherapy and radiation therapy, 4 with chemotherapy alone, and 1 with surgery and radiation therapy. Preoperative KPS scores ranged from 35 to 90 with a median of 90. The average clinical follow-up duration was 274 days (range 7-943 days), with 3 patients not having completed follow-up imaging. The average radiographic follow-up was 214 days (range $25-772$ days). The average number of cryoprobes used for cryoablation was 1.8 (range 1-5), with an average procedure duration of 227 minutes (range 57-483 minutes).

Nine of 14 patients had epidural extension, 7 of whom had postprocedural imaging. There was an average reduction in epidural tumor size; however, this was not found to be significant (paired Student t-test). It was found that a majority of patients (71\%) had stable or reduced radiographic epidural disease burden. See Fig. 2 for an example of a metastatic cervical mass with epidural extension, with successful ablation of the mass and resolution of the epidural compression. Bone remineralization occurred in 5 of 8 patients $(63 \%)$ who had bone included in their ablation volume and postprocedural imaging.

Preoperative VAS pain scores averaged $6.1 \pm 2.9( \pm$ 

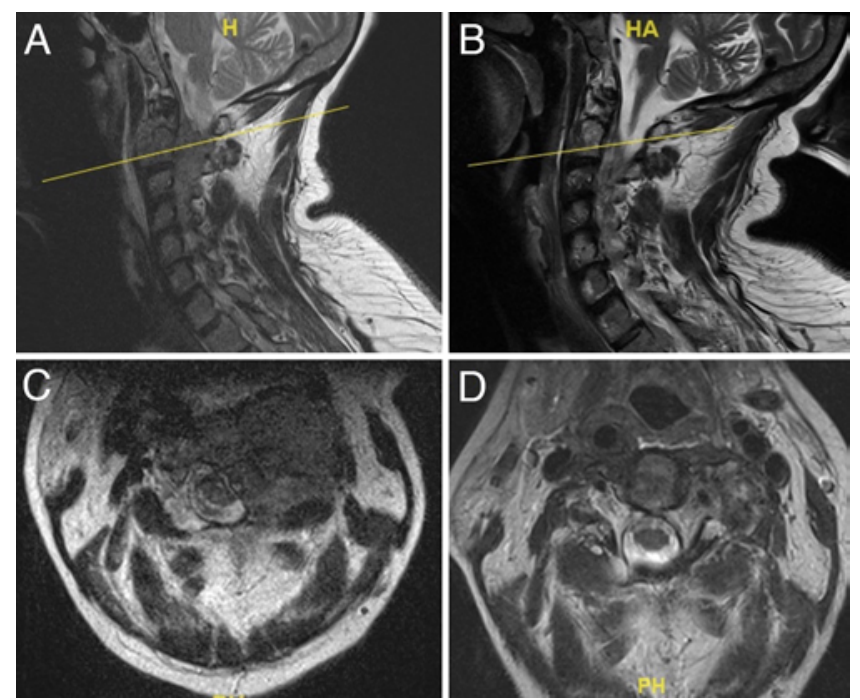

FIG. 2. A: Preoperative sagittal T2-weighted MR image depicting metastatic cervical mass with epidural extension and cord compression. B: Sagittal T2-weighted MR image obtained 5 months postoperatively, demonstrating successful ablation of mass and resolution of cord compression. C: Preoperative axial T2-weighted MR image depicting a metastatic cervical mass with epidural extension and cord compression. D: Axial T2-weighted MR image obtained 5 months postoperatively, demonstrating successful ablation of the mass and resolution of cord compression. Figure is available in color online only.

$\mathrm{SD})$ with a reduction to $3.9 \pm 2.7$ after cryoablation $(\mathrm{p}<$ 0.05 , Wilcoxon signed-rank test; Fig. 3). There were no complications related to the procedure. One patient presented with a preoperative neurological deficit of a right foot drop, which persisted after cryoablation. The median hospital stay was 1 day (range $0-12$ days). In total, 7 of 14 patients $(50 \%)$ went on to have subsequent treatment after cryoablation. Of these, 5 patients were treated with stereotactic radiosurgery, which was deemed safe now that the epidural disease had been treated. The remaining 2 patients went on to have open spine surgery followed by stereotactic surgery. In one of these patients, surgery included tumor debulking and stabilization, which resulted in pain relief. The other patient underwent surgery for tumor debulking followed by stereotactic radiosurgery. Unfortunately, this patient continued to have thoracic radiculopathy with pain that was ultimately managed with nerve blocks, which provided good pain relief. The number of additional spine metastases outside the index level ranged from 0 to 17 with an average of 2.1 metastases.

\section{Discussion}

Symptomatic metastatic spine disease affects approximately $10 \%$ of cancer patients and is projected to increase as patients live longer with control of their primary site of disease..$^{17}$ The neurological, oncological, mechanical, and systemic (NOMS) decision framework is a contemporary organization scheme for treatment decisions regarding metastatic spine disease. ${ }^{11}$ Proponents of this framework advocate the use of stereotactic radiosurgery when possible, limiting surgery to cases with high-grade spinal cord

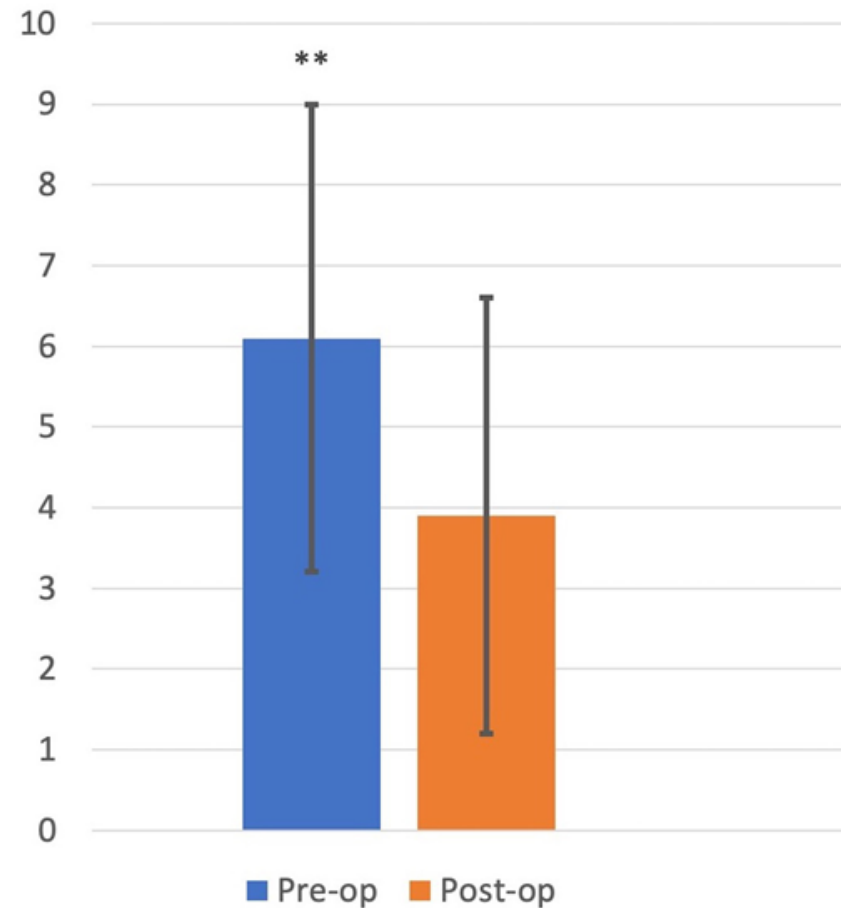

FIG. 3. A Wilcoxon signed-rank test with significant reduction in VAS scores following ablation. Bars denote the mean and error bars the SD. ${ }^{* *} p=0.009$. Figure is available in color online only.

compression (score $>\mathrm{Ib}$ on the ESCC scale), or for spinal fixation in the setting of mechanical instability leading to pain. ${ }^{15}$ In surgical cases, rather than achieving total tumor resection (e.g., spondylectomy), the goals are to decompress the spine with epidural tumor resection and spinal stabilization. With these more modest surgical goals, recovery is faster and patients can go on to receive radiotherapy sooner..$^{15}$ Laufer and colleagues showed a 1-year local progression rate of less than 5\% in a cohort of 189 patients receiving separation surgery followed by stereotactic radiosurgery. ${ }^{10}$

Cryosurgery has been used as a treatment for metastatic bony disease since at least 1964 when a lung cancer patient developed a painful bony metastasis to his humerus. ${ }^{14}$ Despite 2 courses of radiation therapy, he continued to have pain, which resolved completely with a single cryosurgery treatment. Its use has also become popular to treat oligometastatic renal cell cancer. ${ }^{3}$ In one study, the authors found its use to be safe, with only 1 major complication (2\%). They performed a cost-effectiveness analysis and found its use to be valuable as a palliative treatment. ${ }^{3}$ Its efficacy was demonstrated in that only $1 \%$ locally recurred. ${ }^{3}$ In another study analyzing local control of renal cell metastases in 40 patients, cryoablation was found to be safe and effective with a $10 \%$ complication rate and a $4 \%$ local recurrence rate. ${ }^{8}$ Safety and efficacy were demonstrated in at least 2 studies assessing spine cryoablation for vertebral metastases with and without epidural extension. ${ }^{12,21}$ One study comprised 14 patients with 2 transient lower-extremity radiculopathies, local recurrence of $3 \%$, and significant improvements in analgesic usage, ${ }^{21}$ where- 
as the other study only comprised 2 patients but reported no complications and pain reduction with bony regrowth in both patients. ${ }^{12}$ Other studies examining cryoablation more broadly in musculoskeletal metastases have enrolled patients with spinal metastases and have similarly reported favorable safety and efficacy profiles. ${ }^{5,6,18}$ While no studies exist directly comparing cold-based (i.e., cryoablation) and heat-based (i.e., radiofrequency ablation [RFA] and LITT) modalities in the spine, there are retrospective studies on their effectiveness in other regions involving metastatic bony disease. In 2 studies comparing patients who underwent cryoablation or RFA for bony metastatic renal cell cancer, analgesic use intraprocedurally and within the first 24 hours following the procedure was significantly less in the cryoablation group. ${ }^{1,20}$ In addition, hospital length of stay was significantly shorter than in the RFA group. ${ }^{20}$ Another study assessing complications between RFA and cryoablation in renal tumors found no significant difference in complication rates between the two modalities in 533 patients. ${ }^{2}$ The applicability of such a finding to spine metastatic disease is likely limited. Therefore, the main motivation behind the potential use of cryoablation over heat-based modalities would be the evidence suggesting reductions in pain, which is in keeping with palliation as an essential aspect of these treatment modalities. Similar to Tatsui and colleagues, the aims of spinal cryoablation parallel those of sLITT, which include improving recovery time and reducing the delay to initiation of radiation treatment. ${ }^{19}$ In addition, spinal cryoablation offers the benefits of spinal separation to a potentially larger group of patients who may harbor significant comorbidities and are not candidates for traditional open surgery. The cases in this series were deemed not surgical by the multidisciplinary team, and therefore cryoablation was performed.

While sLITT relies on the use of intraoperative fiducial-based navigation to accurately place percutaneous access cannulas, percutaneous access in spinal cryoablation is achieved with CT guidance. This method provides the ability to confirm accurate placement of the cryoprobe prior to transport to the MRI suite, in addition to avoiding the need to make a separate incision to dock the fiducial, which can increase the infection risk, as has been previously reported. ${ }^{19}$ With regard to monitoring the ablation region, the ice ball that is formed during cryoablation shows up as a distinct black ovoid signal void on T2weighted imaging. The images are acquired in less than a minute and offer near-real-time high-resolution visualization of the ablation zone and surrounding anatomy. This is in distinction to sLITT, where thermal mapping is overlaid on previously obtained imaging and predetermined points along the dural edge are monitored rather than a true thin-cut image depicting the actual growth of the ice ball. The mechanisms of cell death in cold- and heat-based modalities have been described, and while coagulation necrosis occurs primarily in both modalities, cryoablation is unique in its ability to induce higher levels of postablative immunogenicity? While we did not study this aspect of cryoablation, in patients with metastatic spine disease, it may provide a positive benefit with regard to inducing the body's immune system to attack other sites of disease, a potential benefit compared with heat-based alternatives.
It is unclear from the small case series that have been reported if the risks of either heat- or cold-based modalities differ; however, Tatsui and colleagues reported a case of spinothalamic dysfunction and T2 signal change following sLITT in the high cervical spine. ${ }^{9}$

In this series, the majority of tumors with epidural extension either remained stable at follow-up imaging or shrank in size. Two patients had interval tumor growth. On review, one of these patients had a vertebral body metastasis with epidural extension, and it appears that the epidural component was not well ablated during the procedure, as the cryoprobe could not be safely positioned to cover this tumor component. The other patient had local progression despite a good ablation zone. Given prior evidence of bone regrowth following cryoablation, this was examined in this cohort of patients. ${ }^{12}$ Indeed, 5 of 8 patients (63\%) with bone that was ablated during the procedure had remineralization of missing bone. In 1 patient it was partial, whereas the remaining 4 had full reconstitution of the ablated bone. All bony regions of the spine showed potential for remineralization, including the pedicle, vertebral body, and lamina. This may be of benefit in patients who do not require instrumentation at presentation, but there is concern for future instability. In 1 patient who presented with tumor involving the vertebral body that underwent cryoablation, regeneration of bone was not seen following ablation, and the patient went on to develop spinal instability and required an instrumented fusion.

Patients tolerated the procedure well with no complications. In addition, half of the patients had a reduction in pain scores soon after ablation, some with marked improvement. This is comparable to the findings with sLITT and in line with previously reported data on cryoablation for vertebral metastases. ${ }^{21}$ Patients generally had short hospital stays and were often discharged home the same day of the procedure.

It is important to note that we do not advocate epidural cryoablation as an alternative to stereotactic radiosurgery or open surgery. Rather, it is a treatment best utilized for patients who may not be amenable to open surgery or for patients with progressive disease despite standard treatment including surgery and stereotactic radiosurgery. In our cohort, half of the patients went on to receive subsequent treatment following cryoablation. The majority of these patients (5/7) received radiosurgery at the level of cryoablation, which became possible following cryosurgery. Two patients went on to undergo surgery, one of whom underwent debulking and stabilization for persistent pain, whereas the other underwent debulking for persistent epidural disease followed by stereotactic radiosurgery.

Several limitations of this study include the small patient cohort, the intermediate follow-up duration, and the lack of randomization and/or a surgical control group. In addition, not all patients received radiation therapy following cryoablation, thus making direct comparisons to traditional separation surgery followed by radiation less clear. However, given the positive safety and efficacy profile of cryoablation, this report lays the preliminary groundwork for a larger study of cryoablation in patients suffering from metastatic spine disease. 


\section{Conclusions}

Cryoablation for metastatic spine disease is a safe and effective way to treat patients who may not otherwise be good candidates for traditional debulking surgery. The use of an advanced imaging suite allows for the percutaneous placement of cryoprobes and active monitoring of the cryotherapy, providing for a short hospital stay and reduced recovery times. Spinal cryoablation can help increase the dose that radiosurgery patients are offered by providing a safe margin of distance between the tumor and neuraxis, in addition to serving as a mechanism to allay spinal pain. Future studies are warranted to better test its applicability in a larger cohort of spinal oncology patients, particularly in those with epidural disease.

\section{References}

1. Allaf ME, Varkarakis IM, Bhayani SB, Inagaki T, Kavoussi LR, Solomon SB: Pain control requirements for percutaneous ablation of renal tumors: cryoablation versus radiofrequency ablation-initial observations. Radiology 237:366-370, 2005

2. Atwell TD, Carter RE, Schmit GD, Carr CM, Boorjian SA, Curry TB, et al: Complications following 573 percutaneous renal radiofrequency and cryoablation procedures. J Vasc Interv Radiol 23:48-54, 2012

3. Bang HJ, Littrup PJ, Goodrich DJ, Currier BP, Aoun HD, Heilbrun LK, et al: Percutaneous cryoablation of metastatic renal cell carcinoma for local tumor control: feasibility, outcomes, and estimated cost-effectiveness for palliation. J Vasc Interv Radiol 23:770-777, 2012

4. Bilsky MH, Laufer I, Fourney DR, Groff M, Schmidt MH, Varga PP, et al: Reliability analysis of the epidural spinal cord compression scale. J Neurosurg Spine 13:324-328, 2010

5. Callstrom MR, Atwell TD, Charboneau JW, Farrell MA, Goetz MP, Rubin J, et al: Painful metastases involving bone: percutaneous image-guided cryoablation-prospective trial interim analysis. Radiology 241:572-580, 2006

6. Callstrom MR, Dupuy DE, Solomon SB, Beres RA, Littrup PJ, Davis KW, et al: Percutaneous image-guided cryoablation of painful metastases involving bone: multicenter trial. Cancer 119:1033-1041, 2013

7. Chu KF, Dupuy DE: Thermal ablation of tumours: biological mechanisms and advances in therapy. Nat Rev Cancer 14:199-208, 2014

8. Gardner CS, Ensor JE, Ahrar K, Huang SY, Sabir SH, Tannir NM, et al: Cryoablation of bone metastases from renal cell carcinoma for local tumor control. J Bone Joint Surg Am 99:1916-1926, 2017

9. Guenette JP, Tuncali K, Himes N, Tatli S, Lee TC: Spine cryoablation: a multimodality image-guided approach for tumors adjacent to major neural elements. AJNR Am J Neuroradiol 37:2396-2399, 2016

10. Laufer I, Iorgulescu JB, Chapman T, Lis E, Shi W, Zhang Z, et al: Local disease control for spinal metastases following "separation surgery" and adjuvant hypofractionated or highdose single-fraction stereotactic radiosurgery: outcome analysis in 186 patients. J Neurosurg Spine 18:207-214, 2013

11. Laufer I, Rubin DG, Lis E, Cox BW, Stubblefield MD, Yamada Y, et al: The NOMS framework: approach to the treatment of spinal metastatic tumors. Oncologist 18:744-751, 2013

12. Lee TC, Guenette JP, Moses ZB, Chi JH: MRI-guided cryoablation of epidural malignancies in the spinal canal resulting in neural decompression and regrowth of bone. AJR Am J Roentgenol 212:205-208, 2019
13. Maraka S, Asmaro K, Walbert T, Lee I: Cerebral edema induced by laser interstitial thermal therapy and radiotherapy in close succession in patients with brain tumor. Lasers Surg Med 50:917-923, 2018

14. Marcove RC, Miller TR: The treatment of primary and metastatic localized bone tumors by cryosurgery. Surg Clin North Am 49:421-430, 1969

15. Moussazadeh N, Laufer I, Yamada Y, Bilsky MH: Separation surgery for spinal metastases: effect of spinal radiosurgery on surgical treatment goals. Cancer Contr 21:168-174, 2014

16. Patchell RA, Tibbs PA, Regine WF, Payne R, Saris S, Kryscio RJ, et al: Direct decompressive surgical resection in the treatment of spinal cord compression caused by metastatic cancer: a randomised trial. Lancet 366:643-648, 2005

17. Perrin RG, Laxton AW: Metastatic spine disease: epidemiology, pathophysiology, and evaluation of patients. Neurosurg Clin N Am 15:365-373, 2004

18. Prologo JD, Passalacqua M, Patel I, Bohnert N, Corn DJ: Image-guided cryoablation for the treatment of painful musculoskeletal metastatic disease: a single-center experience. Skeletal Radiol 43:1551-1559, 2014

19. Tatsui CE, Stafford RJ, Li J, Sellin JN, Amini B, Rao G, et al: Utilization of laser interstitial thermotherapy guided by real-time thermal MRI as an alternative to separation surgery in the management of spinal metastasis. J Neurosurg Spine 23:400-411, 2015

20. Thacker PG, Callstrom MR, Curry TB, Mandrekar JN, Atwell TD, Goetz MP, et al: Palliation of painful metastatic disease involving bone with imaging-guided treatment: comparison of patients' immediate response to radiofrequency ablation and cryoablation. AJR Am J Roentgenol 197:510_ 515,2011

21. Tomasian A, Wallace A, Northrup B, Hillen TJ, Jennings JW: Spine cryoablation: pain palliation and local tumor control for vertebral metastases. AJNR Am J Neuroradiol 37:189_ 195,2016

\section{Disclosures}

The authors report no conflict of interest concerning the materials or methods used in this study or the findings specified in this paper.

\section{Author Contributions}

Conception and design: Chi, Lee, Guenette. Acquisition of data: all authors. Analysis and interpretation of data: Moses, Guenette. Drafting the article: Chi, Moses, Lee, Guenette. Critically revising the article: Moses, Lee, Huang, Guenette. Reviewed submitted version of manuscript: Lee, Guenette. Approved the final version of the manuscript on behalf of all authors: Chi. Statistical analysis: Moses. Study supervision: Chi, Lee.

\section{Supplemental Information}

Previous Presentations

Some of the data were presented at the AANS/CNS Section on Disorders of the Spine and Peripheral Nerves Spine Summit, Miami, Florida, March 16, 2019.

\section{Correspondence}

John H. Chi: Brigham and Women's Hospital, Boston, MA. jchi@ bwh.harvard.edu. 